\title{
Artroplastia invertida lateralizada para artropatía del manguito rotador en pacientes $>60$ años. Resultados y complicaciones a corto plazo
}

\author{
Diego Gómez, Tamara Dainotto, Gonzalo Viollaz, Gustavo J. Teruya, Alejandro Tedeschi, Rafael Durán, Álvaro Muratore \\ Equipo de Miembro Superior, Servicio de Ortopedia y Traumatología, Hospital Británico de Buenos Aires, Ciudad Autónoma de Buenos \\ Aires, Argentina
}

\section{RESUMEN}

Objetivo: Comunicar los resultados y las complicaciones a corto plazo de la artropatía del manguito rotador tratada con artroplastia invertida lateralizada en pacientes $>60$ años. Materiales y Métodos: Se identificaron retrospectivamente las artropatías del manguito rotador tratadas con prótesis invertida lateralizada entre 2015 y 2019. Criterios de inclusión: edad >60 años, adecuada función deltoidea y seguimiento mínimo de 24 meses. Antes de la cirugía y después, se registraron el rango de movilidad activa, y los puntajes de Constant-Murley y de la escala analógica visual para dolor. En las radiografías, se determinaron los grados de artrosis glenohumeral, de muescas escapulares y de aflojamiento de la prótesis. Se documentaron las complicaciones, la satisfacción con el procedimiento y la supervivencia del implante. Resultados: La serie incluyó 40 hombros operados en 38 pacientes (edad promedio 72 años, rango 61-91), con un seguimiento promedio de 25 meses. La elevación anterior aumentó de $82,63^{\circ}$ a $136,71^{\circ}$ ( $p<0,001$ ); la rotación externa, de $16,59^{\circ}$ a $32,11^{\circ}$ ( $\left.p<0,001\right)$ y la rotación interna, de L5 a L3 ( $\left.p<0,001\right)$. El puntaje de Constant-Murley ascendió de 28,25 a 69,97 ( $p<0,001$ ) y el de dolor disminuyó de 8,34 precirugía a 1,26 poscirugía ( $p<0,001$ ). La tasa de complicaciones fue del 12,5\% y la supervivencia del implante, del 97,4\%. Conclusiones: La artroplastia invertida lateralizada es una excelente alternativa quirúrgica para la artropatía del manguito rotador en pacientes $>60$ años, los resultados son satisfactorios, la tasa de complicaciones es aceptable y la supervivencia del implante es del $97,4 \%$.

Palabras clave: Artroplastia invertida lateralizada; artropatía; manguito rotador; prótesis invertida.

Nivel de Evidencia: IV

\section{Reverse Total Shoulder Arthroplasty in Patients $>60$ Years of Age With Rotator Cuff Tear Arthropathy. Short-Term Outcomes and Complications}

\begin{abstract}
Background: The purpose of this study is to show the short-term results and complications in a series of patients $>60$ who underwent a reverse arthroplasty for the treatment of rotator cuff arthropathy in a high complexity hospital. Materials and Methods: We retrospectively analyzed our Service's database to identify patients with rotator cuff arthropathy who had undergone a reverse shoulder arthroplasty between 2015 and 2019. Inclusion criteria: 60 years of age or older, functional deltoid, at least one year of follow-up. The range of motion was measured pre and postoperatively in flexion, external rotation, and internal rotation, as well as Constant score and visual analog scale for pain. The grade of arthritis, scapular notching, and prosthetic loosening was radiographically assessed. We identified complications, satisfaction, and prosthesis survivorship. Results: Forty shoulders in 38 patients of 72 years of age on average (61-91) underwent reverse shoulder arthroplasty, with 25 months of follow-up. Flexion increased from $82.86^{\circ}$ to $136.71^{\circ}(p<0.001)$, external rotation went from $16.59^{\circ}$ to $32.11^{\circ}(p<0.001)$, and internal rotation from L5 to $L 3(p<0.001)$. Constant score rose from 28.25 to $69.97(p<0.001)$ and visual analog scale for pain decreased from 8.34 to 1.26 $(p<0.001)$. The complication rate was $12.5 \%$ and a $97.4 \%$ prosthesis survivorship was registered. Conclusions: We believe that reverse shoulder arthroplasty represents an excellent option in the treatment of rotator cuff arthropathy in patients older than 60 in view of the satisfactory functional outcomes with an acceptable complication rate.
\end{abstract}

Key words: Lateralized reverse arthroplasty; rotator cuff arthropathy; shoulder arthroplasty.

Level of Evidence: IV

Recibido el 25-9-2020. Aceptado luego de la evaluación el 1-4-2021 • Dr. DIEGO GÓMEZ • drdjg@ hotmail.com (ID https://orcid.org/0000-0003-0258-6802

Cómo citar este artículo: Gómez D, Dainotto T, Viollaz G, Teruya GJ, Tedeschi A, Durán R, Muratore Á. Artroplastia invertida lateralizada para artropatía del manguito rotador en pacientes >60 años. Resultados y complicaciones a corto plazo. Rev Asoc Argent Ortop Traumatol 2021;86(6):780-789. https://doi.org/10.15417/issn. 1852-7434.2021.86.6.1209 


\section{INTRODUCCIÓN}

La artropatía del manguito rotador (AMR), descrita inicialmente por Charles Neer, ${ }^{1}$ es un cuadro desafiante al que nos enfrentamos los cirujanos de hombro. Se estima que un $4 \%$ de las lesiones del manguito rotador son masivas y evolucionan con atrofia e infiltración grasa muscular, artrosis glenohumeral, dolor y limitación de la movilidad..$^{2,3}$ La AMR representa el estadio final de esta condición, en la que se intensifica la sintomatología y compromete severamente la realización de las actividades cotidianas. ${ }^{4}$

Entre las alternativas quirúrgicas para tratar la AMR, la artroplastia invertida (AI) se ha convertido en el patrón de referencia, ${ }^{5}$ logra una mejoría promedio en la escala de Constant-Murley ${ }^{6}$ de $45-55$ puntos $^{7}$ y valores de elevación activa posoperatoria de $138^{\circ} .^{7-12}$ Sin embargo, pese a que es un procedimiento con resultados predecibles, la tasa de complicaciones varía entre el $17 \%$ y el $20 \%$, y la de reoperación oscila entre el 3,5\% y el 11,9\%. ${ }^{7,8,13}$ Las principales causas son luxación de la prótesis $(1,2-4,7 \%)$, infección (3-3,8\%), aflojamiento del componente glenoideo $(1,8-8,8 \%),{ }^{14}$ fracturas periprotésicas $(1,1-1,5 \%)$ y lesiones neurológicas $(0,4-1,2 \%) .{ }^{8,13}$

El diseño original de la prótesis invertida, ideado por Grammont, consistió en medializar y distalizar el centro de rotación de la articulación glenohumeral para potenciar el brazo de palanca deltoideo. ${ }^{15}$ Estos implantes provocaron algunas complicaciones específicas, como la generación de muescas escapulares, ${ }^{15}$ que disminuyeron gracias a las AI lateralizadas, aportando, a su vez, una menor incidencia de luxación de la prótesis y un mayor rango de movilidad en las rotaciones. ${ }^{11}$

En la actualidad, es escasa la bibliografía nacional sobre el tratamiento de las AMR con AI. ${ }^{7}$ Por este motivo, un estudio clínico sobre los resultados y las complicaciones en el medio local puede proveer la información necesaria para la planificación y la toma de decisiones, con el fin de mejorar la calidad de la atención de este grupo específico de pacientes.

El objetivo del estudio fue evaluar los resultados y las complicaciones a corto plazo derivados de la AI lateralizada en pacientes $>60$ años con AMR en un centro de alta complejidad de la Ciudad Autónoma de Buenos Aires.

\section{MATERIALES Y MÉTODOS}

Se analizó retrospectivamente la base de datos de nuestro Servicio con el objetivo de identificar los casos de AMR tratados con una AI lateralizada por un mismo equipo quirúrgico, entre enero de 2015 y enero de 2019.

Se aplicaron los siguientes criterios de inclusión: 1) artropatía por síndrome del manguito rotador (Hamada $\geq 3$ ), 2) edad $>60$ años, 3) deltoides funcionante, 4) seguimiento mínimo de 24 meses y 5) uso de prótesis con diseño lateralizado.

Se excluyó a pacientes con secuelas postraumáticas, artritis reumatoide, cuadros neurológicos (enfermedad de Parkinson, esclerosis múltiple).

\section{Técnica quirúrgica}

Con el paciente en posición de silla de playa, bajo anestesia plexual, se realiza un abordaje deltopectoral, con tenotomía insercional completa del subescapular y sección de la cápsula articular. Se luxa la cabeza humeral en posición de rotación externa máxima y se procede a la osteotomía humeral con $20^{\circ}$ de retroversión y $135^{\circ}$ de inclinación. Posteriormente, se trabaja el canal humeral con raspas crecientes hasta obtener una sensación de fricción cortical. La raspa de mayor calibre utilizada se deja colocada para proteger el húmero proximal durante el tiempo glenoideo.

Al realizar la exposición glenoidea, se resecan los osteofitos y el labrum degenerativo, optimizando la visión de la superficie articular. Luego, se lleva a cabo el fresado del cartílago articular conservando el mayor stock óseo posible. La metaglena se implanta proyectando una inclinación $<10^{\circ}$ con versión neutra. La fijación definitiva se efectúa con dos tornillos de compresión de 5,5 $\mathrm{mm}$. Utilizamos glenoesferas de $36 \mathrm{~mm}$ para mujeres y hombres pequeños, y reservamos la glenoesfera de $39 \mathrm{~mm}$ para hombres corpulentos.

El componente humeral definitivo se coloca de forma no cementada, siempre que la calidad ósea metafisaria lo permita. A continuación, se selecciona el tamaño del inserto de polietileno definitivo para obtener una construcción protésica balanceada y estable. Se realiza la reinserción del subescapular con puntos transóseos.

Se inmoviliza a todos los pacientes por 30 días con un cabestrillo tipo Velpeau, los ejercicios de movilidad pasiva comienzan a la semana de la cirugía y la rehabilitación kinésica, a la cuarta semana. 


\section{Evaluación clínica}

La evaluación y el registro de los datos estuvieron a cargo de un residente de ortopedia y traumatología que no formó parte de los procedimientos quirúrgicos (TD). Los datos preoperatorios se obtuvieron, de manera retrospectiva, revisando las historias clínicas de consultorios. Se registraron los rangos de movilidad preoperatorio y posoperatorio en el último control. La movilidad activa se evaluó con goniómetro en elevación anterior, rotación externa con el miembro en aducción y rotación interna con la mano en la espalda, se registró como máximo nivel el segmento alcanzado por el pulgar de acuerdo con la descripción de Greene y Heckman. ${ }^{16}$ La función muscular del deltoides la definió el cirujano tratante, según la escala motora de Daniels, considerando deltoides funcionante al equiparar con un valor de M5. ${ }^{17}$

La función se evaluó con la escala de Constant-Murley ${ }^{6}$ y el dolor, con la escala analógica visual, ${ }^{18}$ antes del procedimiento y después de él.

Además, se evaluó el grado de satisfacción posoperatoria mediante dos preguntas: 1. ¿Qué grado de satisfacción siente respecto al procedimiento realizado? Las posibles respuestas eran tres: insatisfecho, satisfecho, muy satisfecho. 2. ¿Recomendaría esta intervención a una persona conocida con la misma patología que usted a modo de tratamiento? En este caso, las opciones eran sí o no.

Se identificaron las complicaciones intraoperatorias y posoperatorias en los registros de las historias clínicas de internación, los protocolos quirúrgicos y los controles por consultorios externos. Se consideró que una complicación era menor cuando no alteró el resultado final ni fue necesaria una reoperación; en caso contrario, se la consideró una complicación mayor.

Se definió como revisión a cualquier procedimiento quirúrgico, cerrado o abierto, relacionado con la artroplastia.

\section{Evaluación radiográfica}

Se utilizó la clasificación de Hamada ${ }^{19}$ para determinar el grado de artrosis glenohumeral en las radiografías preoperatorias de hombro de frente y axial de escápula. En todos los casos, se completó el estudio con una tomografía computarizada para ejecutar la planificación prequirúrgica, jerarquizando la presencia de defectos óseos, y las variaciones de inclinación y retroversión glenoidea.

La valoración radiográfica posoperatoria se realizó a los 10 días, 6 semanas, 12 semanas, 24 semanas y luego anualmente. En las últimas radiografías tomadas, se evaluó la presencia de muescas escapulares según la clasificación de Sirveaux, ${ }^{11}$ de signos de aflojamiento glenoideo, según Cuff y cols., ${ }^{3}$ de cambios en el posicionamiento del componente respecto a las radiografías anteriores y de líneas de radiolucencia alrededor del vástago según Sperling ${ }^{20}$.

\section{Análisis estadístico}

Las variables continuas se expresan en porcentajes como frecuencias absolutas y relativas. Se aplicó la prueba t de Student para datos preoperatorios y posoperatorios apareados con distribución normal focalizando la magnitud de las diferencias de media. Se empleó la prueba de rangos con signo de Wilcoxon en la comparación de medianas con distribución no normal. Se consideró estadísticamente significativo un valor $\mathrm{p}<0,05$. Se utilizó el programa $\mathrm{IBM}^{\circledR}$ SPSS $^{\circledR}$ Statistics versión 25.

\section{RESULTADOS}

Se analizaron 44 AI lateralizadas, y la serie quedó conformada por 40 AI realizadas en 38 pacientes que tenían una edad promedio de 72 años (rango 61-91) y cumplían con los criterios de inclusión. El seguimiento promedio fue de 25 meses (rango 2-4 años). Se excluyeron cuatro AI, porque el seguimiento era $<2$ años. Se utilizó la prótesis ARROW® (FH Orthopaedics, Mulhouse, Francia) en todos los casos. La glenoesfera fue de $36 \mathrm{~mm}$ en 37 hombros y de $39 \mathrm{~mm}$ en los tres restantes. La dimensión la definió el cirujano a cargo de acuerdo con el tamaño del paciente. Las características demográficas de la muestra se detallan en la Tabla 1.

Se registró una elevación anterior posoperatoria de $136,71^{\circ}$ (rango $30-160^{\circ}$ ), con un incremento de $54,08^{\circ}$ (rango $80-170^{\circ}$ ) respecto del valor inicial $\left(\mathrm{p}<0,001\right.$ ); una rotación externa preoperatoria que aumentó de $16,59^{\circ}$ (rango $\left.0-60^{\circ}\right)$ a $32,11^{\circ}\left(\right.$ rango $\left.5-70^{\circ}\right)(\mathrm{p}<0,001)$ y una rotación interna posoperatoria hasta L3 en promedio, lo que representó una mejoría de dos segmentos corporales $(\mathrm{p}<0,001)$ (Figura 1). 
Tabla 1. Características demográficas

\begin{tabular}{|c|c|}
\hline Total & 40 hombros \\
\hline Edad & 72 años (rango 61-91) \\
\hline Seguimiento & 25 meses (rango $2-4$ años) \\
\hline Sexo & \\
\hline Masculino & $11(29 \%)$ \\
\hline Femenino & $27(71 \%)$ \\
\hline
\end{tabular}

\begin{tabular}{l|l} 
Lado & \\
Derecho & 25 \\
Izquierdo & 13
\end{tabular}

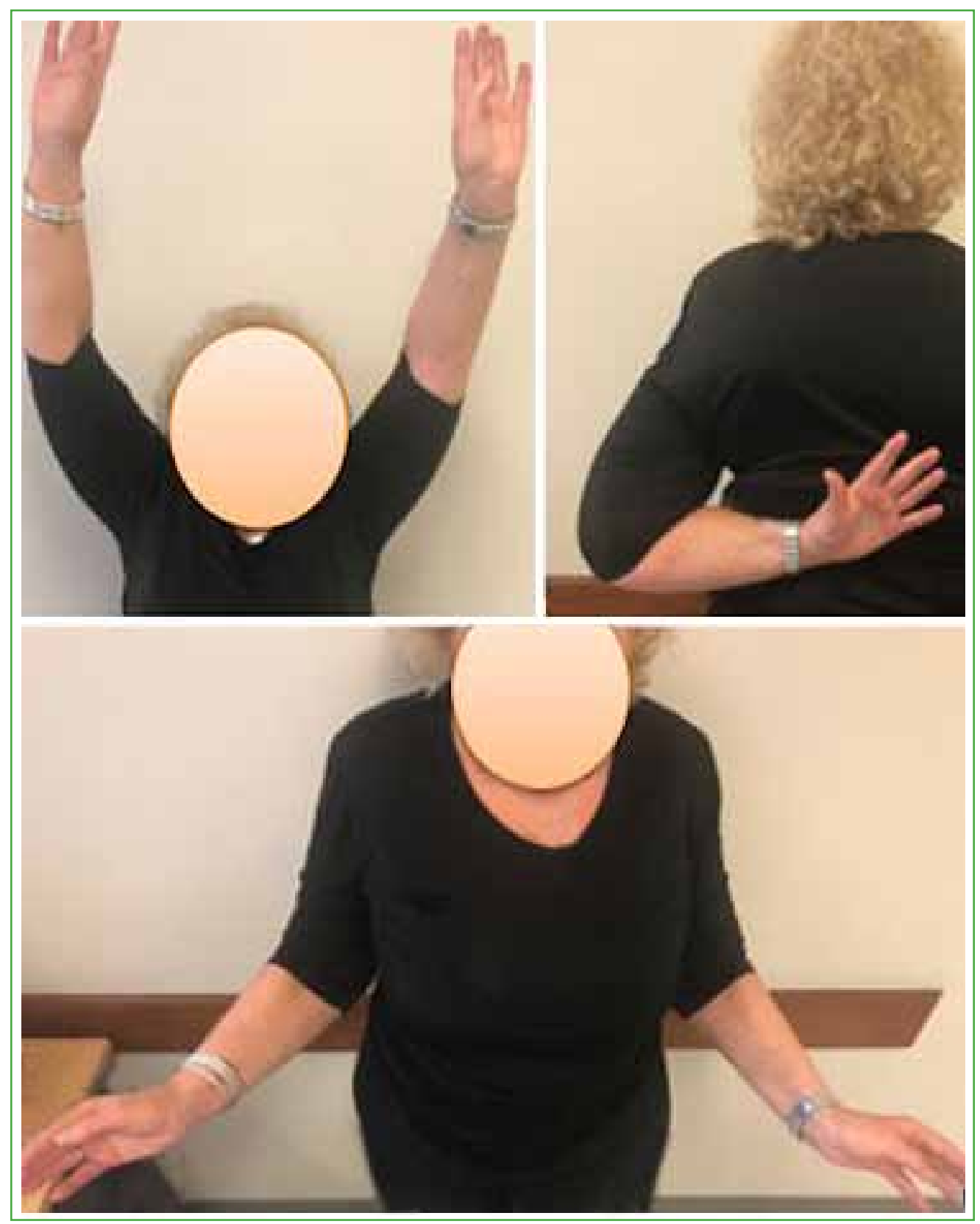

Figura 1. Control al año y medio de la artroplastia invertida lateralizada de hombro izquierdo. Rango de movilidad de elevación anterior $170^{\circ}$, rotación externa $45^{\circ}$ y rotación interna hasta $\mathrm{T} 10$. 
El puntaje promedio de la escala de Constant-Murley mejoró de 28,25 (rango 12-40) a 69,97 (rango 44-83) en el último seguimiento $(\mathrm{p}<0,001)$ y el de la escala analógica visual, de 8,34 (rango 7-10) antes de la cirugía a 1,26 (rango 0-6) en el posoperatorio ( $\mathrm{p}<0,001)$ (Tabla 2).

Tabla 2. Resultados funcionales

\begin{tabular}{|l|c|c|}
\hline & Preoperatorio & Posoperatorio \\
\hline Escala de Constant-Murley & 28,2 & $70(\mathrm{p}<0,001)$ \\
\hline Escala analógica visual & 8,4 & $1,2(\mathrm{p}<0,001)$ \\
\hline Elevación anterior & $82,6^{\circ}$ & $136,7^{\circ}(\mathrm{p}<0,001)$ \\
\hline Rotación externa & $16,6^{\circ}$ & $32,1^{\circ}(\mathrm{p}<0,001)$ \\
\hline Rotación interna & $\mathrm{L} 5$ & $\mathrm{~L} 3$ \\
\hline
\end{tabular}

La tasa de complicaciones fue del 12,5\% (5 casos). Cuatro fueron intraoperatorias ( 2 complicaciones menores y 2 mayores). Dos complicaciones menores (5\%) fueron una fractura glenoidea que requirió la colocación de una glena de revisión en el mismo acto quirúrgico, sin que esto afectara el resultado obtenido. Una paciente sufrió dos complicaciones mayores. Se trató de uno de los primeros casos de nuestro Centro, en el comienzo de la curva de aprendizaje. La paciente sufrió una fractura periprotésica intraoperatoria de la diáfisis humeral, sintetizada con cerclajes y tratada con un brace, que evolucionó con neuropraxia del nervio radial y resolución parcial. Otra paciente evolucionó con un cuadro de insuficiencia acromial, debido a una fragilización acromial preoperatoria que repercutió en el rango de movilidad (Figura 2).

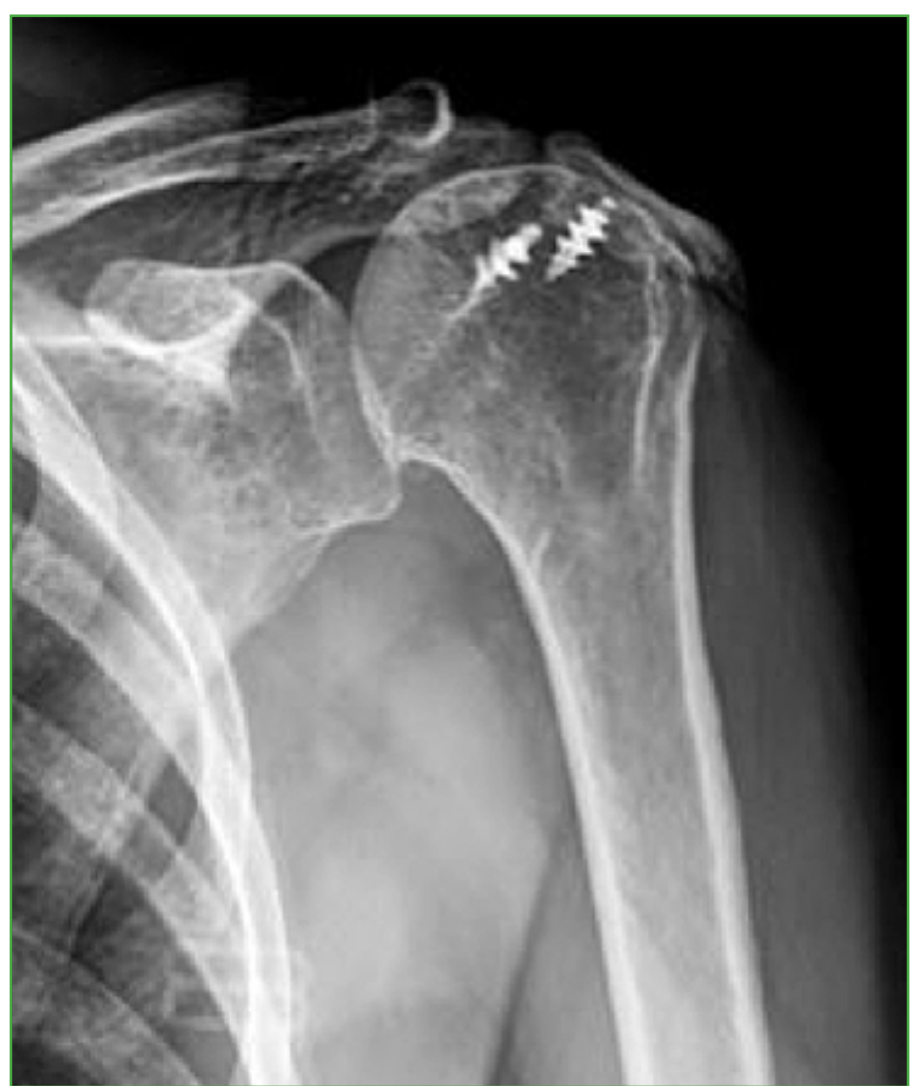

Figura 2. Radiografía de hombro derecho de frente. Se observa la fragilización acromial. 
En las radiografías, se detectó que dos pacientes tenían muescas glenoideas grado I (Figura 3). No hubo casos de aflojamiento del componente humeral, sí se registró como única complicación mayor posoperatoria el aflojamiento mecánico del componente glenoideo en un paciente a los 14 meses de la cirugía inicial; en este caso, se procedió a la revisión con colocación de una glena de revisión (Figura 4). La tasa de supervivencia del implante fue del 97,5\%.

En cuanto a la satisfacción con la cirugía, 28 pacientes (74\%) refirieron estar muy satisfechos; ocho, satisfechos (21\%). Solo dos (5\%) se manifestaron insatisfechos con el resultado: la paciente que sufrió las dos complicaciones mayores registradas y la otra paciente con fragilización acromial preoperatoria. Asimismo, el 95\% afirmó que recomendaría la $\mathrm{AI}$ a una persona conocida que sufra AMR.

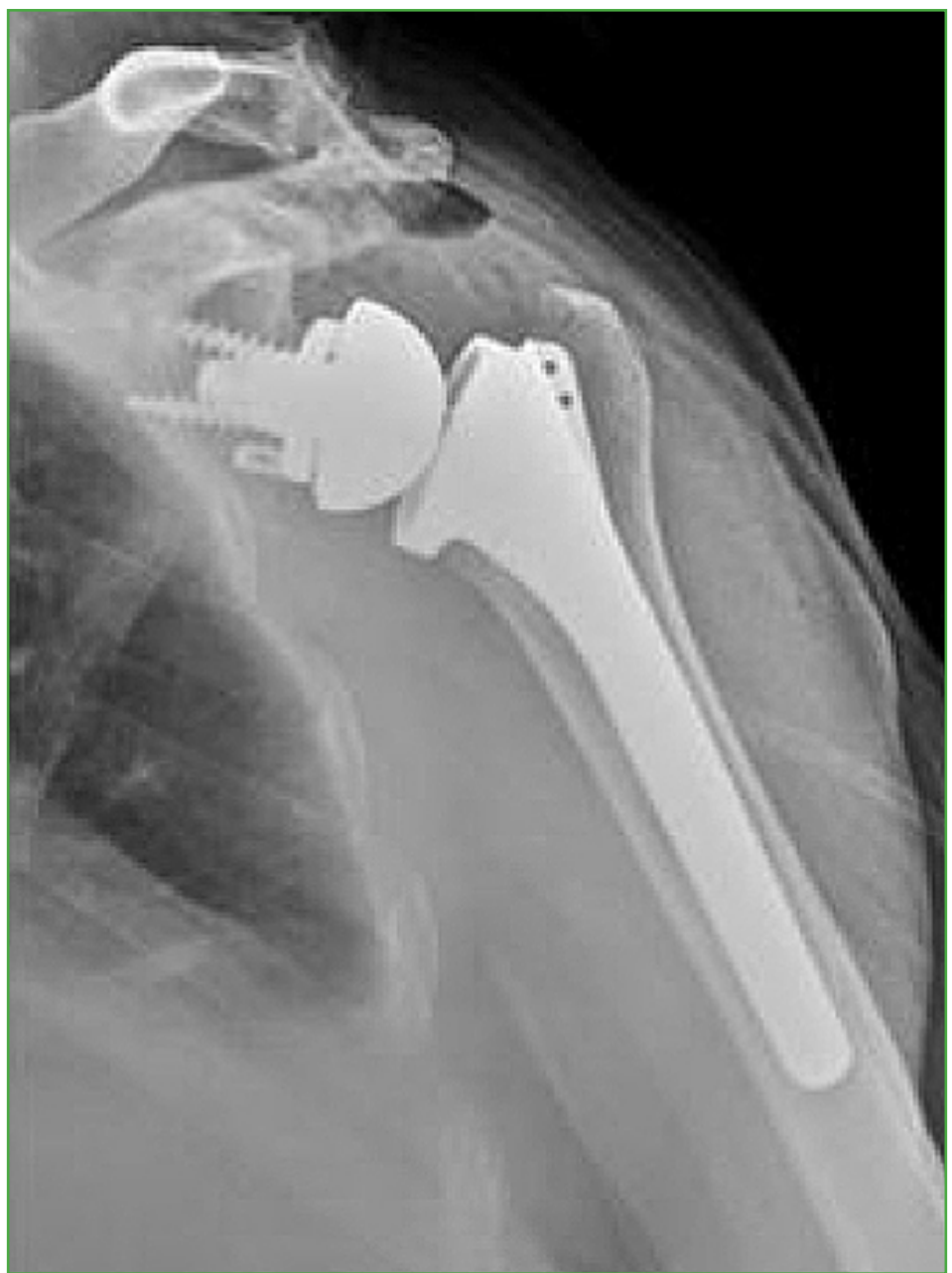

Figura 3. Radiografía de hombro izquierdo de frente. Se observa una muesca escapular grado I. 


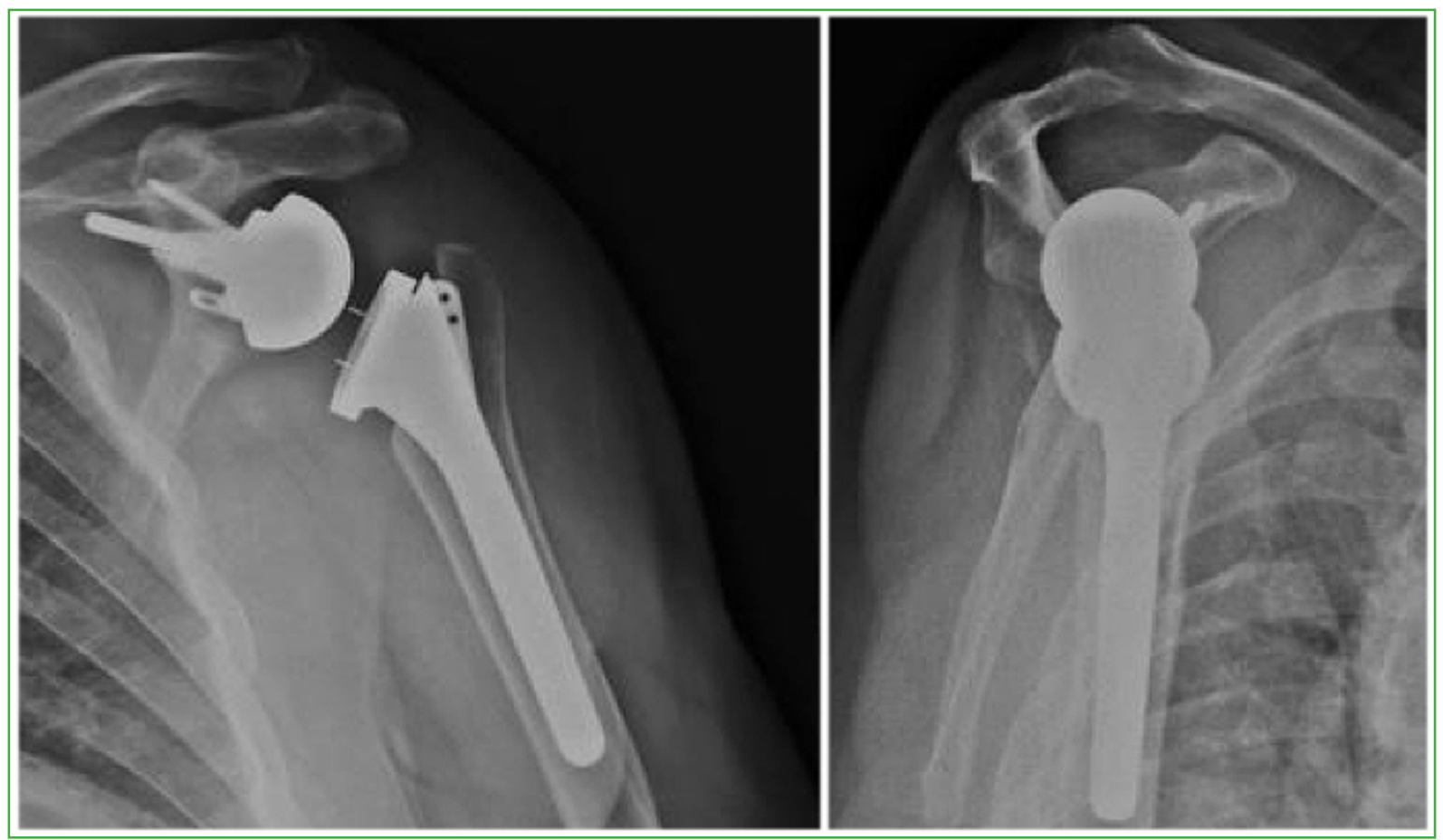

Figura 4. Radiografías de hombro izquierdo, de frente y de perfil, a los seis meses de la revisión del componente glenoideo.

\section{DISCUSIÓN}

El hallazgo más importante del estudio es la tasa de satisfacción del 95\%, con una supervivencia del implante del $97,5 \%$ y una tasa global de complicaciones del $12,5 \%$.

La alta tasa de supervivencia coincide con los resultados hallados en la bibliografía. Con la revisión como punto de corte, los estudios comunican tasas de supervivencia de aproximadamente el $95 \%$ a los cinco años ${ }^{21}$ y del $91 \%$ a los 10 años $^{22}$. Como las series publicadas muestran sus registros a partir de los cinco años de seguimiento, a diferencia de los dos años mínimos de nuestra serie, nuestros resultados pueden verse afectados en la comparación con la bibliografía. Este estudio, al contar con un seguimiento mínimo de dos años, puede subestimar la tasa de complicaciones futuras observadas en estudios con un seguimiento más prolongado.

Los valores medios obtenidos fueron $136,71^{\circ}$ para la elevación anterior y $32,11^{\circ}$ para la rotación externa, que concuerdan con los de otros autores, como Nolan y cols., y Boileau y cols., en 71 y 21 casos, respectivamente (en ambos, la elevación anterior obtenida fue de $121^{\circ}$ y la rotación externa, de $15^{\circ}$ y $11^{\circ}$, respectivamente) con prótesis medializadas, e inclusive alcanzando valores más altos de rotación externa en comparación..$^{12,23}$ Asimismo, en una serie de 34 pacientes, Wiater y cols. informan una elevación anterior de $131^{\circ}$ y una rotación externa de $26,6^{\circ}$ en promedio, empleando prótesis lateralizadas. ${ }^{9}$

El puntaje de la escala de Constant-Murley resultante fue de 69,97, acorde al indicado por otros autores, que oscila entre 59 puntos, según Boileau y cols., en 45 pacientes, controlados durante 40 meses, y 86 puntos obtenidos por Bacle y cols., con un implante lateralizado a 10 años de seguimiento. ${ }^{10,12}$ Asimismo, el puntaje posoperatorio de la escala analógica visual fue de 1,26, similar a los publicados., ${ }^{9,1223}$

La tasa de complicaciones $(12,5 \%)$ fue inferior a las de otras publicaciones que varían entre el $15 \%$ y el $22 \% \cdot{ }^{8,11,12,23}$ Se observaron solo dos muescas glenoideas grado I, y no hubo episodios de luxación de la prótesis. Atribuimos la baja tasa de muescas e inestabilidad al uso de implantes lateralizados, los cuales, a diferencia de los medializados tipo Grammont, permiten un mayor rango de movilidad libre de topes óseos. ${ }^{24}$ Valenti y cols., utilizando el mismo implante, no comunicaron episodios de luxación de la prótesis en 76 hombros. ${ }^{24}$ Esto se justifica tanto por la tensión magnificada de las partes blandas remanentes, como por la concavidad pronunciada del inserto de polietileno. ${ }^{24}$ Además, la reparación del subescapular podría representar un factor de protección respecto a las luxaciones de la prótesis. Sin embargo, esto sigue siendo un tema controvertido. ${ }^{25}$ 
Se produjo una fractura de la diáfisis humeral al realizar la reducción definitiva en uno de nuestros primeros pacientes, quien además evolucionó con parálisis del nervio radial. En una serie de 203 AI, García-Fernández y cols. describieron tres casos de fracturas humerales, de las cuales solo una era diafisaria y ocurrió durante el fresado medular en la revisión de una hemiartroplastia. ${ }^{26}$ Por su parte, Chuinard y cols. informaron 26 fracturas humerales intraquirúrgicas en $457 \mathrm{AI}$. En esta serie, 25 ocurrieron en revisiones y una se produjo en una artroplastia primaria, durante el labrado del canal medular. ${ }^{27}$ Por otra parte, la lesión directa del nervio radial durante el procedimiento quirúrgico de $\mathrm{AI}$ es infrecuente según la bibliografía. Consideramos que estas infrecuentes complicaciones neurológicas, tal como postulan Boileau y cols., se deben a la elongación que sufren las ramas terminales del plexo braquial durante el acto quirúrgico por el alargamiento de la longitud del húmero. ${ }^{12}$

Entre las fracturas intraoperatorias de glena, en una revisión sistemática, Zumstein y cols. comunicaron una prevalencia del $0,9 \%$ en 782 hombros, que estuvieron principalmente relacionadas con el fresado articular. ${ }^{13}$ Sirveaux y cols. tuvieron una prevalencia del 1,5\% en $1953 \mathrm{AI}$, el riesgo fue mayor en ascenso severos de la cabeza humeral. ${ }^{11}$ El 5\% de fracturas glenoideas en nuestra serie resulta levemente superior al reportado por Sirveaux y cols. ${ }^{11}$ Esto se justifica, en parte, por la curva de aprendizaje con este implante, que además se caracteriza por una resección ósea glenoidea más agresiva. Un paciente tuvo un aflojamiento mecánico del componente glenoideo a los 14 meses de la cirugía; esta complicación ocurre en el 1,8-8,8\% de los casos. ${ }^{14,28}$ Pese a que aún no se han identificado factores de riesgo asociados, en su serie de 202 hombros tratados con prótesis lateralizada y una incidencia del 1,2\% de esta complicación en cirugías primarias, Bitzer y cols. identificaron como factores de riesgo el uso de injerto óseo para suplir defectos glenoideos y la fijación del baseplate con tornillos no bloqueados. ${ }^{14}$ En nuestro paciente, el aflojamiento se debió a una toma insuficiente de los tornillos glenoideos, que fue tratado luego con una metaglena de revisión; el paciente cursa el sexto mes posoperatorio sin complicaciones agregadas.

En esta serie, una paciente presentaba fragilización acromial preoperatoria, lo cual repercutió desfavorablemente en el rango de movilidad máximo a la elevación anterior, era solo de $90^{\circ}$ en el último control posoperatorio. Este resultado se puede explicar teniendo en cuenta que la inserción acromial del deltoides es un elemento clave en la función de la AI ${ }^{15}$ El descenso y la medialización del centro de rotación glenohumeral con el objetivo de restaurar la tensión deltoidea son fundamentales para mejorar la elevación anterior activa. Se ha propuesto que la patología acromial preoperatoria puede comprometer la función deltoidea y afectar el correcto funcionamiento de la prótesis, a diferencia de Walch y cols., quienes evaluaron a 28 pacientes con AMR tratada mediante AI y con patología acromial, y compararon los resultados funcionales con los obtenidos en pacientes sin lesión acromial. Estos autores no hallaron diferencias en el rango de movilidad posoperatorio y el puntaje de la escala de Constant-Murley. ${ }^{28}$

Es preciso considerar algunas limitaciones antes de analizar estos datos, teniendo en cuenta las limitaciones inherentes a la metodología descriptiva retrospectiva. Asimismo, el hecho de no contar con un grupo de control imposibilitó comparar los resultados de prótesis lateralizada con los obtenidos con implantes medializados. El tiempo mínimo de seguimiento de dos años no permite identificar fehacientemente la tasa de complicaciones a largo plazo, esto justifica la baja frecuencia de complicaciones registradas en esta serie. Entre las fortalezas, destacamos que se trata del primer informe nacional con este tipo de implantes, que incluye una cantidad considerable de pacientes que cuentan con un registro preciso de los resultados funcionales y las complicaciones posoperatorias inmediatas, así como con la evaluación de la satisfacción de los pacientes.

Queda pendiente para una próxima línea de trabajo una serie comparativa con prótesis medializadas y un seguimiento más prolongado.

\section{CONCLUSIONES}

Consideramos la AI lateralizada como una excelente alternativa quirúrgica para la AMR en pacientes $>60$ años, proporciona resultados clínicos y radiográficos satisfactorios, con una tasa de complicaciones aceptable y una supervivencia del implante del 97,4\% a los 25 meses de seguimiento promedio. Creemos que nuestro estudio representa un aporte a la bibliografía nacional, pues proporciona un mejor fundamento para la planificación quirúrgica y la toma de decisiones a fin de mejorar la calidad de vida de los pacientes que sufren esta enfermedad.

Conflicto de intereses: Los autores no declaran conflictos de intereses. 
ORCID de T. Dainotto: https://orcid.org/0000-0002-6645-9928 ORCID de G. Viollaz: https://orcid.org/0000-0002-4573-883X ORCID de G. J. Teruya: https://orcid.org/0000-0001-7342-1859
ORCID de A. Tedeschi: https://orcid.org/0000-0001-5704-3122 ORCID de R. Durán: https://orcid.org/0000-0002-8789-322 ORCID de Á. Muratore: https://orcid.org/0000-0001-7540-7137

\section{BIBLIOGRAFÍA}

1. Neer CS, Craig E V, Fukuda H. Cuff-tear arthropathy. J Bone Joint Surg 1983;65(9):1232-44. PMID: 6654936

2. Boileau P, Watkinson DJ, Hatzidakis AM, Balg F. Grammont reverse prosthesis: design, rationale, and biomechanics. J Shoulder Elbow Surg 2005;14(1 Suppl.):S147-S61. https://doi.org/10.1016/j.jse.2009.03.011

3. Cuff D, Pupello D, Virani N, Levy J, Frankle M. Reverse shoulder arthroplasty for the treatment of rotator cuff deficiency. J Bone Joint Surg Am 2008;90(6):1244-51. https://doi.org/10.2106/JBJS.G.00775

4. Giráldez EÁ, Gavilán F. Efectividad y seguridad de las prótesis invertidas de hombro en artropatía del manguito rotador. Revisión sistemática de la literatura y criterios de uso adecuado.En: Informes, estudios e investigación. Ministerio de Sanidad, Servicios Sociales e Igualdad. Serie: Informes de Evaluación de Tecnologías Sanitarias. Agencia de Evaluación de Tecnologías Sanitarias de Andalucía. Disponible en: https://www.aetsa.org/download/publicaciones/antiguas/AETSA_2011_12_ProtesisInvertida.pdf

5. Chawla H, Gamradt S. Reverse total shoulder arthroplasty: technique, decision-making and exposure tips. Curr Rev Musculoskelet Med 2020;13(2):180-5. https://doi.org/10.1007/s12178-020-09613-3

6. Constant CR, Murley AHG. A clinical method of functional assessment of the shoulder. Clin Orthop Relat Res 1987(214):160-4. PMID: 3791738

7. Bertona Altieri BA, Rossi L, Oñativia JI, Tanoira I, Bongiovanni SL, Maignon G, et al. Resultados a corto plazo de la prótesis inversa de hombro según la patología. Rev Asoc Argentina Ortop Traumatol 2017;82(4):278-86. https://doi.org/10.15417/683

8. Petrillo S, Longo UG, Papalia R, Denaro V. Reverse shoulder arthroplasty for massive irreparable rotator cuff tears and cuff tear arthropathy: a systematic review. Musculoskelet Surg 2017;101(2):105-12.

https://doi.org/10.1007/s12306-017-0474-z

9. Wiater JM, Moravek JE, Budge MD, Koueiter DM, Marcantonio D, Wiater BP. Clinical and radiographic results of cementless reverse total shoulder arthroplasty: A comparative study with 2 to 5years of follow-up. J Shoulder Elbow Surg 2014;23(8):1208-14. http://doi.org/10.1016/j.jse.2013.11.032

10. Bacle G, Nové-Josserand L, Garaud P, Walch G. Long-term outcomes of reverse total shoulder arthroplasty. J Bone Joint Surg Am 2017;99(6):454-61. http://doi.org/10.2106/JBJS.16.00223

11. Sirveaux F, Favard L, Oudet D, Huquet D, Walch G, Molé D. Grammont inverted total shoulder arthroplasty in the treatment of glenohumeral osteoarthritis with massive rupture of the cuff. Results of a multicentre study of 80 shoulders. J Bone Joint Surg Br 2004;86(3):388-95. https://doi.org/10.1302/0301-620x.86b3.14024

12. Boileau P, Watkinson D, Hatzidakis AM, Hovorka I. Neer Award 2005: The Grammont reverse shoulder prosthesis: Results in cuff tear arthritis, fracture sequelae, and revision arthroplasty. J Shoulder Elbow Surg 2006;15:527-40. https://doi.org/10.1016/j.jse.2006.01.003

13. Zumstein M, Pinedo M, Old J, Boileau P. Problems, complications, reoperations, and revisions in reverse total shoulder arthroplasty: A systematic review. J Shoulder Elbow Surg 2011;20(1):146-57. https://doi.org/10.1016/j.jse.2010.08.001

14. Bitzer A, Rojas J, Patten I, Joseph J, McFarland E. Incidence and risk factors for aseptic baseplate loosening of reverse total shoulder arthroplasty. J Shoulder Elbow Surg 2018;(12):2145-52. https://doi.org/10.1016/j.jse.2018.05.034

15. Grammont PM, Baulot E. Delta shoulder prosthesis for rotator cuff rupture. Orthopedics 1992;16(1):65-8. PMID: 8421661

16. Greene WB, Heckman JD. The shoulder. En: The clinical measurement of joint motion. Rosemont: American Academy Orthopaedic Surgeons; 1994:15-26.

17. Hislop HJ, Avers D, Brown M. Daniels y Worthingham. Técnicas de balance muscular, 9a ed. Philadelphia: Elsevier; 2014.

18. Huskisson EC. Measurement for pain. Lancet 1974;2(7889):1127-31. https://doi.org/10.1016/s0140-6736(74)90884-8

19. Hamada K, Fukuda H, Mikasa M, Kobayashi Y. Roentgenographic findings in massive rotator cuff tears. A longterm observation. Clin Orthop Relat Res 1990;(254):92-6. https://doi.org/10.1097/00003086-199005000-00014 
20. Sperling JW, Cofield RH, O'Driscoll SW, Torchia ME, Rowland CM. Radiographic assessment of ingrowth total shoulder arthroplasty. J Shoulder Elbow Surg 2000;9(6):507-13. https://doi.org/10.1067/mse.2000.109384

21. Cuff D, Clark R, Pupello D, Frankle M. Reverse shoulder arthroplasty for the treatment of rotator cuff deficiency: a concise follow-up, at a minimum of five years, of a previous report. J Bone Joint Surg Am 2012;94(21):1996-2000. https://doi.org/10.2106/JBJS.K.01206

22. Guery J, Favard L, Sirveaux F, Oudet D, Mole D, Walch G. Reverse total shoulder arthroplasty. Survivorship analysis of eighty replacements followed for five to ten years. J Bone Joint Surg Am 2006;(8):1742-7. https://doi.org/10.2106/JBJS.E.00851

23. Nolan BM, Ankerson E, Wiater MJ. Reverse total shoulder arthroplasty improves function in cuff tear arthropathy. Clin Orthop Relat Res 2011;469(9):2476-82. https://doi.org/10.1007/s11999-010-1683-Z

24. Valenti P, Sauzières P, Katz D, Kalouche I, Kilinc AS. Do less medialized reverse shoulder prostheses increase motion and reduce notching? Clin Orthop Relat Res 2011;469(9):2550-7. https://doi.org/10.1007/s11999-011-1844-8

25. Cheung E, Sarkissian E, Sox-Harris A, Comer G, Saleh J, Diaz R, Costouros J. Instability after reverse total shoulder Arthroplasty. J Shoulder Elbow Surg 2018,(11):1946-52. https://doi.org/10.1016/j.jse.2018.04.015

26. García-Fernández C, Lópiz-Morales Y, Rodríguez A, López-Durán L, Martínez FM. Periprosthetic humeral fractures associated with reverse total shoulder arthroplasty: incidence and management. Int Orthop 2015;(10):1965-9. https://doi.org/10.1007/s00264-015-2972-7

27. Chuinard C, Trojani C, Brassart N BP. Humeral problems in reverse shoulder arthroplasty. En: Walch G, Boileau P, Molé D, et al (eds.) Reverse shoulder arthroplasty. Clinical results, complications, revisions. Montpellier, France: Sauramps Medical; 2006:275-88. https://doi.org/10.1007/978-3-030-19285-3

28. Walch G, Mottier F, Wall B, Boileau P, Molé D, Favard L. Acromial insufficiency in reverse shoulder arthroplasties. J Shoulder Elbow Surg 2009;18(3):495-50. https://doi.org/10.1016/j.jse.2008.12.002 